hep-ph/9806404

DO-TH 98/07

WUE-ITP-98-019

June 1998

\title{
Dynamical Parton Distributions Revisited
}

\author{
M. Glück ${ }^{1}$, E. Reya ${ }^{1}$ and A. Vogt ${ }^{2}$ \\ ${ }^{1}$ Institut für Physik, Universität Dortmund \\ D-44221 Dortmund, Germany \\ ${ }^{2}$ Institut für Theoretische Physik, Universität Würzburg \\ Am Hubland, D-97074 Würzburg, Germany
}

\begin{abstract}
Dynamical parton densities, generated radiatively from valence-like inputs at some low resolution scale, are confronted with recent small- $x$ data on deep inelastic and other hard scattering processes. It is shown that within theoretical uncertainties our previous (1994) dynamical/radiative parton distributions are compatible with most recent data and still applicable within the restricted accuracy margins of the presently available next-to-leading order calculations. Due to recent high precision measurements we also present an updated, more accurate, version of our (valence-like) dynamical input distributions. Furthermore, our perturbatively stable parameter-free dynamical predictions are extended to the extremely small $-x$ region, $10^{-8} \lesssim x \lesssim 10^{-5}$, relevant to questions concerning ultra-high-energy cosmic ray and neutrino astronomy.
\end{abstract}




\section{Introduction}

The guiding physical idea underlying the dynamical (radiative) parton model is that the steep behavior of the momentum distributions $x f\left(x, Q^{2}\right)(f=q, \bar{q}, g)$ at $x \lesssim 10^{-2}$, $Q^{2} \gtrsim 1 \mathrm{GeV}^{2}$ is a purely perturbative phenomenon. In fact, in [1, 2, 3] non-steep (valencelike) initial distributions $x f\left(x, \mu^{2}\right)$ at some low scale $\mu \approx 0.6 \mathrm{GeV}$ were suggested in order to predict, purely dynamically, the rise of $x \bar{q}$ and $x g$ in the above range of $x$ and $Q^{2}$. This prediction was subsequently confirmed by the measurements of $F_{2}^{p}\left(x, Q^{2}\right)$ and $x g\left(x, Q^{2}\right)$ at HERA [4, 5]. As was stated in [2, 3], the available pre-HERA data at $x>10^{-2}$ utilized to fix the valence-like input distributions still allowed for a slight, typically $\mathrm{O}(10 \%)$, variation (increase) of $\mu$ which did not affect $F_{2}\left(x>10^{-2}, Q^{2}\right)$ but resulted in an about $10 \%(20 \%)$ uncertainty of the radiative predictions at $x=10^{-3}\left(10^{-4}\right)$. Hence the $\mathrm{O}(10 \%)$ discrepancies between the distributions of [3] and recent precision measurements at HERA [6, 7, 8] are obviously neither unexpected, nor do they invalidate the notion of a radiative, i.e. dynamical, origin for the steep rise at $x \lesssim 10^{-2}$ and $Q^{2} \gtrsim$ $1 \mathrm{GeV}^{2}$. Indeed, a fine-tuning of $\mu$ and/or $f\left(x, \mu^{2}\right)$ was always understood [2, 3] to be necessary in due course.

Given the accuracy of recent HERA small- $x$ data, as well as new large $-x$ constraints, mainly on the flavor decomposition of the quark sea, it is now appropriate to perform an update of our previous dynamical parton distributions and to follow the effects of these fine-tunings on the predictions for $F_{2}\left(x, Q^{2}\right), g\left(x, Q^{2}\right)$ and other relevant deep inelastic observables.

Section 2 will be devoted mainly to a discussion concerning the small- $x$ implications of the recent $\mathrm{H} 1$ and ZEUS high precision data [6, 7, 8] on $F_{2}^{p}\left(x, Q^{2}\right)$ and $g\left(x, Q^{2}\right)$ and how they (slightly) modify our previous [3] valence-like LO/NLO input gluon and sea distributions. Further data on $F_{2}^{c}\left(x, Q^{2}\right)$ and fixed-target results on $F_{2}^{p}, F_{2}^{n} / F_{2}^{p}$ and $d_{v} / u_{v}$, as well as data on $p p(d) \rightarrow \mu^{+} \mu^{-} X$ and $p \bar{p} \rightarrow W^{ \pm} X$ asymmetries relevant for fixing and testing our $g, u_{v}, d_{v}, \bar{d}-\bar{u}$ and $u / d$ densities are presented and compared with the present update and with our previous GRV(94) results in Section 3. Furthermore, we also extend here our perturbatively stable and parameter-free dynamical small- $x$ predictions to the ultra small- $x$ region $\left(10^{-8} \lesssim x \lesssim 10^{-5}\right)$, relevant to questions concerning ultra-high-energy cosmic ray and neutrino astronomy. Finally, in Section 4 we present a brief summary and a general discussion concerning the status of the dynamical (radiative) parton model in the light of present and future data and its application in theoretical NLO analyses. 


\section{Consequences of recent data on $F_{2}\left(x, Q^{2}\right)$ and parton densities}

As stated in the Introduction we intend to study the implications of modifications of our original GRV(94) input [3]. We shall proceed in a stepwise manner and begin with a mere modification of the input scale $\mu^{2}$ keeping everything else unchanged. The results shown in Figs. 1 and 2 illustrate the point that the previously noted [2, 3] uncertainty in $\mu^{2}$ can accommodate the main discrepancies between [3] and the recent data [6, 7, 8] on $F_{2}\left(x, Q^{2}\right)$ and the experimentally extracted $x g\left(x, Q^{2}\right)$. It should be noted that our present new (modified) analysis yields results which lie in between the two curves shown in Fig. 1 as will be discussed and illustrated in Fig. 3. Our new results (solid curve) for $x g\left(x, Q^{2}\right)$ at $Q^{2}=20 \mathrm{GeV}^{2}$ are shown as well in Fig. 2 where they practically coincide with the one of GRV(94) with $\mu_{\mathrm{NLO}}$ increased by $10 \%$ (dotted curve). The fact that the somewhat too steep GRV(94) predictions for $F_{2}\left(x, Q^{2}\right)$ and $x g\left(x, Q^{2}\right)$ at, say, $x=10^{-4}$ can both be corrected with the new slightly modified valence-like input constitutes a nontrivial confirmation of the radiative model.

Let us now turn to the update of our LO/NLO GRV(94) densities which consists of a fine-tuning of the valence-like input densities for $f\left(x, \mu^{2}\right)$ as well as of the input scale $\mu$. This fine-tuning of the input results mainly from new HERA data [6, 7, 8] on $F_{2}\left(x, Q^{2}\right)$ and to a certain extent also from new large $-x$ results (such as asymmetry measurements of Drell-Yan dilepton production in $p p$ and $p d$ collisions [9, 10]) discussed in more detail in Section 3. For the running coupling $\alpha_{s}\left(Q^{2}\right)$ at the next-to-leading order (NLO) we have utilized the exact numerical solution! of

$$
\frac{d \alpha_{s}\left(Q^{2}\right)}{d \ln \left(Q^{2}\right)}=-\frac{\beta_{0}}{4 \pi} \alpha_{s}^{2}\left(Q^{2}\right)-\frac{\beta_{1}}{16 \pi^{2}} \alpha_{s}^{3}\left(Q^{2}\right)
$$

with $\beta_{0}=11-2 f / 3$ and $\beta_{1}=102-38 f / 3$, which is nowadays used for NLO analyses [6, 8, 11, 12, 13] since it is stable against yet higher order contributions, thus being more appropriate in the low $Q^{2}$ region. This is in contrast to using [1], 2, [3] the approximate

\footnotetext{
${ }^{1}$ Alternatively, the exact solution can be written implicitly

$$
\ln \frac{Q^{2}}{\tilde{\Lambda}_{\overline{\mathrm{MS}}}^{2}}=\frac{4 \pi}{\beta_{0} \alpha_{s}\left(Q^{2}\right)}-\frac{\beta_{1}}{\beta_{0}^{2}} \ln \left[\frac{4 \pi}{\beta_{0} \alpha_{s}\left(Q^{2}\right)}+\frac{\beta_{1}}{\beta_{0}^{2}}\right],
$$

and our new NLO results correspond, for $\alpha_{s}\left(M_{Z}^{2}\right)=0.114$, to the following values of $\tilde{\Lambda}_{\frac{(f)}{\mathrm{MS}}}: \tilde{\Lambda}_{\frac{(3,4,5,6)}{\mathrm{MS}}}=$ 299.4, 246, 167.7, $67.8 \mathrm{MeV}$.
} 
solution

$$
\frac{\alpha_{s}\left(Q^{2}\right)}{4 \pi} \simeq \frac{1}{\beta_{0} \ln \left(Q^{2} / \Lambda^{2}\right)}-\frac{\beta_{1}}{\beta_{0}^{3}} \frac{\ln \ln \left(Q^{2} / \Lambda^{2}\right)}{\left[\ln \left(Q^{2} / \Lambda^{2}\right)\right]^{2}}
$$

which is sufficiently accurate for $Q^{2} \gtrsim m_{c}^{2}$. Here, $\Lambda$ refers in NLO to $\Lambda \equiv \Lambda_{\overline{\mathrm{MS}}}$ and in LO $\left(\beta_{1}=0\right)$ to $\Lambda \equiv \Lambda_{\mathrm{LO}}$. We have chosen $\alpha_{s}\left(M_{Z}^{2}\right)=0.114$ for obtaining our exact numerical NLO solutions from (1) for $Q^{2} \geq \mu_{\mathrm{NLO}}^{2}$. This choice, which is slightly preferred in our present analysis, agrees with the average value of the space-like momentum-transfer measurements [13, 14, 15], $\alpha_{s}\left(M_{Z}^{2}\right)=0.114 \pm 0.005$. The statistically dominating timelike $e^{+} e^{-}$LEP $Z^{0}$-data imply a somewhat larger 'world average' [13, 14] of $\alpha_{s}\left(M_{Z}^{2}\right)=$ $0.118 \pm 0.005$ with an error which is to some extent uncertain and debatable. It should be kept in mind, however, that LEP data ( $Z^{0}$ hadronic decays) allow [16] also for a much smaller strong coupling, $\alpha_{s}\left(M_{Z}^{2}\right)=0.101 \pm 0.013$.

Should significantly higher values of $\alpha_{s}$, e.g. $\alpha_{s}\left(M_{Z}^{2}\right)=0.118$, turn out to be undebated and everywhere unique, then our input scale $\mu$ will obviously increase closer to $1 \mathrm{GeV}$ which compels one to give up the strict valence-like sea input $x \bar{q}\left(x, \mu^{2}\right)$, but not the valence-like gluon input $x g\left(x, \mu^{2}\right)$, in order to reproduce all $F_{2}^{p}\left(x, Q^{2}\right)$ data at $Q^{2} \gtrsim$ $1 \mathrm{GeV}^{2}$ [1]. Alternatively one may keep the valence-like sea input $x \bar{q}\left(x, \mu^{2}\right)$, also for $\alpha_{s}\left(M_{Z}^{2}\right)=0.118$, as a parameter-free seed for the small- $x$ structure of $F_{2}\left(x, Q^{2}>\mu^{2}\right)$, in case one intends to predict and explain the HERA-data merely above $Q^{2} \simeq 3 \mathrm{GeV}^{2} \llbracket 12$.

Furthermore the conventional approximate formula (2), being sufficiently reliable for $Q^{2} \gtrsim m_{c}^{2}$, corresponds in our case to $\Lambda \frac{(4,5,6)}{\mathrm{MS}}=257,173.4,68.1 \mathrm{MeV}$ which reproduces the exact solutions to even better than $0.5 \%$ for $Q^{2} \geq 5 \mathrm{GeV}^{2}$. Our LO results correspond to $\Lambda_{\mathrm{LO}}^{(3,4,5,6)}=204,175,132,66.5 \mathrm{MeV}$ which leads to the (theoretically less relevant) value of $\alpha_{s}^{\mathrm{LO}}\left(M_{Z}^{2}\right)=0.125$. In both cases we have used for the $\alpha_{s}$ matchings

$$
m_{c}=1.4 \mathrm{GeV}, \quad m_{b}=4.5 \mathrm{GeV}, \quad m_{t}=175 \mathrm{GeV} .
$$

These masses are used in all our subsequent LO and NLO analyses for heavy quark production. In particular the value of $m_{c}$, which is slightly lower than the one previously employed in [1, 2, 3], is favored.

The free parameters of the non-singlet input densities $u_{v}, d_{v}, \Delta \equiv \bar{d}-\bar{u}$ and of the valence-like singlet input distributions $\bar{d}+\bar{u}$ and $g$ at $Q^{2}=\mu^{2}$ have been fixed using the following data sets: the published 1994 and 1995 HERA $F_{2}^{p}$ results [6, [] for $Q^{2} \geq 2 \mathrm{GeV}^{2}$; the fixed target $F_{2}^{p}$ data of SLAC [17], BCDMS [18, 19], NMC [20] and E665 [21] subject to the standard cuts $Q^{2} \geq 4 \mathrm{GeV}^{2}$ and $W^{2}=Q^{2}\left(\frac{1}{x}-1\right)+m_{p}^{2} \geq 10 \mathrm{GeV}^{2}$; the 
structure function ratios $F_{2}^{n} / F_{2}^{p}$ [22, 23], together with the $u_{v} / d_{v}$ results from $\stackrel{(-)}{\nu} p(d)$ DIS [24; the Drell-Yan muon-pair production data of E605 25] for $d^{2} \sigma^{p N} / d x_{F} d M_{\mu^{+} \mu^{-}}$and of NA51 [9] and E866 [10] for the cross section ration $\sigma^{p d} / \sigma^{p p}$. The input fit parameters/normalizations of $u_{v}$ and $d_{v}$ are further constrained by $\int_{0}^{1} u_{v} d x=2$ and $\int_{0}^{1} d_{v} d x=1$, and the ones of the gluon density by the energy-momentum conservation relation

$$
\int_{0}^{1} x\left[u_{v}\left(x, \mu^{2}\right)+d_{v}\left(x, \mu^{2}\right)+2 \bar{u}\left(x, \mu^{2}\right)+2 \bar{d}\left(x, \mu^{2}\right)+g\left(x, \mu^{2}\right)\right] d x=1 .
$$

The resulting $\mathrm{LO}$ input distributions at $Q^{2}=\mu_{\mathrm{LO}}^{2}=0.26 \mathrm{GeV}^{2}$ are then given by

$$
\begin{aligned}
x u_{v}\left(x, \mu_{\mathrm{LO}}^{2}\right) & =1.239 x^{0.48}(1-x)^{2.72}(1-1.8 \sqrt{x}+9.5 x) \\
x d_{v}\left(x, \mu_{\mathrm{LO}}^{2}\right) & =0.614(1-x)^{0.9} x u_{v}\left(x, \mu_{\mathrm{LO}}^{2}\right) \\
x \Delta\left(x, \mu_{\mathrm{LO}}^{2}\right) & =0.23 x^{0.48}(1-x)^{11.3}(1-12.0 \sqrt{x}+50.9 x) \\
x(\bar{u}+\bar{d})\left(x, \mu_{\mathrm{LO}}^{2}\right) & =1.52 x^{0.15}(1-x)^{9.1}(1-3.6 \sqrt{x}+7.8 x) \\
x g\left(x, \mu_{\mathrm{LO}}^{2}\right) & =17.47 x^{1.6}(1-x)^{3.8} \\
x s\left(x, \mu_{\mathrm{LO}}^{2}\right) & =x \bar{s}\left(x, \mu_{\mathrm{LO}}^{2}\right)=0
\end{aligned}
$$

where $\Delta \equiv \bar{d}-\bar{u}$. It is interesting to note that $\mu_{\mathrm{LO}}=2.5 \Lambda_{\mathrm{LO}}^{(3)}=2.9 \Lambda_{\mathrm{LO}}^{(4)}$ and $\alpha_{s}^{\mathrm{LO}}\left(\mu_{\mathrm{LO}}^{2}\right) / \pi=$ 0.24. The corresponding $\mathrm{NLO}(\overline{\mathrm{MS}})$ input at $Q^{2}=\mu_{\mathrm{NLO}}^{2}=0.40 \mathrm{GeV}^{2}$ is U

$$
\begin{aligned}
x u_{v}\left(x, \mu_{\mathrm{NLO}}^{2}\right) & =0.632 x^{0.43}(1-x)^{3.09}(1+18.2 x) \\
x d_{v}\left(x, \mu_{\mathrm{NLO}}^{2}\right) & =0.624(1-x)^{1.0} x u_{v}\left(x, \mu_{\mathrm{NLO}}^{2}\right) \\
x \Delta\left(x, \mu_{\mathrm{NLO}}^{2}\right) & =0.20 x^{0.43}(1-x)^{12.4}(1-13.3 \sqrt{x}+60.0 x) \\
x(\bar{u}+\bar{d})\left(x, \mu_{\mathrm{NLO}}^{2}\right) & =1.24 x^{0.20}(1-x)^{8.5}(1-2.3 \sqrt{x}+5.7 x) \\
x g\left(x, \mu_{\mathrm{NLO}}^{2}\right) & =20.80 x^{1.6}(1-x)^{4.1} \\
x s\left(x, \mu_{\mathrm{NLO}}^{2}\right) & =x \bar{s}\left(x, \mu_{\mathrm{NLO}}^{2}\right)=0 .
\end{aligned}
$$

Note again that $\mu_{\mathrm{NLO}}=2.1 \tilde{\Lambda}_{\frac{\mathrm{MS}}{(3)}}=2.6 \tilde{\Lambda}_{\overline{\mathrm{MS}}}^{(4)}$ and $\alpha_{s}\left(\mu_{\mathrm{NLO}}^{2}\right) / \pi=0.18$, and that there is a correlation between the chosen value of $\alpha_{s}\left(M_{Z}^{2}\right)$ and the resulting value for $\mu_{\mathrm{NLO}}$, which increases with $\alpha_{s}\left(M_{Z}^{2}\right)$ as already discussed above. Note that our obtained value for $\mu_{\mathrm{NLO}}$

\footnotetext{
${ }^{2}$ The power $a_{\text {sea }}$ of the valence-like LO/NLO sea input densities $x(\bar{u}+\bar{d})\left(x, \mu^{2}\right) \sim x^{a_{\text {sea }}}$, as $x \rightarrow 0$, depends strongly on the choice of $\mu$, i.e. on the chosen value for $\alpha_{s}\left(M_{Z}^{2}\right)$ or, equivalently, on $\Lambda^{(3)}$. We would obtain almost equally agreeable results if we continue to use the same $\alpha_{s}\left(Q^{2}\right)$ as in our previous analyses [1, 2, 3] which was based on the approximate evolution formula (2). Matching the approximate $\mathrm{NLO}(\overline{\mathrm{MS}}) \alpha_{s}\left(Q^{2}\right)$ to the exact numerical solution of eq. (1) at, say, $Q^{2}=3 \mathrm{GeV}^{2}$ results in $\alpha_{s}\left(M_{Z}^{2}\right)=0.110$. As discussed above, this $\alpha_{s}$ value lies at the lower end of the presently allowed experimental bounds. In this case one obtains $\mu_{\mathrm{NLO}}^{2} \simeq 0.30 \mathrm{GeV}^{2}$ and $a_{\mathrm{sea}}^{\mathrm{NLO}} \simeq 0.35$, instead of 0.20 in eq. (6).
} 
would have been larger if we had used the (inappropriate) approximate formula (2) instead of the exact solution of (1). It should be furthermore noted that we have chosen, as previously [3], a vanishing strange sea at the input scale $\mu$ in order to comply with experimental indications [26, 27] of an SU(3)-broken sea. This choice is also supported and slightly favored by our input fits and compares well [28] with recent measurements [27 of $s\left(x, Q^{2}>\mu^{2}\right)$. Our $s\left(x, Q^{2}\right)$ is thus generated purely dynamically (radiatively) and therefore constitutes, for the time being, an absolute, i.e. parameter-free prediction. If future experiments may require a finite strange sea input, then our present results for $s\left(x, Q^{2}\right)$ have to be interpreted as an absolute lower bound for the strange sea. Furthermore, the charm contribution $F_{2}^{c}\left(x, Q^{2}\right)$ to $F_{2}$ is provided by the perturbatively stable 29] fixed-order perturbation theory. In LO it derives from the well known photon-gluon fusion process [3] $\gamma^{*} g \rightarrow c \bar{c}$. For the NLO calculations we employ the $O\left(\alpha_{s}^{2}\right)$ coefficient functions of [30] as conveniently parametrized in [31]. In both cases we use $m_{c}=1.4 \mathrm{GeV}$ as given in (3) and choose the factorization and renormalization scales to equal $4 m_{c}^{2}$. The bottom contribution $F_{2}^{b}$ is marginal, reaching at most 1 to $2 \%$.

Our resulting small- $x$ predictions for $F_{2}^{p}$ are shown in Fig. 3 and for $x g\left(x, Q^{2}=20\right.$ $\mathrm{GeV}^{2}$ ) by the solid curve in Fig. 2. These results are not too different from our NLO GRV(94) expectations [3] when comparing them with the NLO(94) results in Figs. 1 and 2. Moreover, the NLO results in Fig. 3 are favored over the LO ones in the small $Q^{2}$ region, $Q^{2} \lesssim 3 \mathrm{GeV}^{2}$ (which might indicate that future NNLO contributions could even improve our NLO results in the small $Q^{2}$ region around $1 \mathrm{GeV}^{2}$ ). Furthermore, their dependence on choosing different factorization scales [14, 32], instead of $\mu_{F}^{2}=Q^{2}$, is obviously weaker than in LO. Nevertheless, the present LO/NLO stability is even better than for the GRV(94) $F_{2}$-predictions. The LO and NLO input densities in eqs. (5) and (6) are shown in Fig. 4 and compared with the NLO ones of GRV(94) [3] as well as with their evolutions to $Q^{2}=5 \mathrm{GeV}^{2}$. The appropriate valence-like NLO input $F_{2}^{p}\left(x, \mu_{\mathrm{NLO}}^{2}\right)$, which eventually vanishes as $x \rightarrow 0$, is also shown in the lower right corner of Fig. 3 . This illustrates very clearly that the predictions at $x<10^{-2}$ and $Q^{2}>\mu_{\mathrm{NLO}}^{2}$ are of a purely dynamical origin, in particular the increase of $F_{2}$ with $x$ as $x \rightarrow 0$ is due to the non-vanishing input at $x \gtrsim 10^{-2}$. Nevertheless, as evident from Fig. 3, our predictions for $Q^{2} \lesssim 1 \mathrm{GeV}^{2}$ fall below the data in the (very) small-x region. This is not unexpected for leading twist-2 results, since nonperturbative (higher twist) contributions to $F_{2}\left(x, Q^{2}\right)$ have eventually to become dominant for decreasing values of $Q^{2}$. 
It is also interesting to note that the total momentum fractions carried by the NLO input distributions at $Q^{2}=\mu^{2}$ in Fig. 4 amount to 56, 30 and $14 \%$ for valence, gluon and sea densities, respectively, which are very similar in LO and similar to our GRV(94) results [3].

Further typical small- $x$ predictions for $x g\left(x, Q^{2}\right)$ and $x \bar{u}\left(x, Q^{2}\right)$ are shown in Fig. 5 together with their respective inputs at $Q^{2}=\mu^{2}$ which become, particularly for the gluon input, vanishingly small at $x<10^{-2}$. This illustrates again the purely dynamical origin of the small- $x$ structure of gluon and sea quark densities at $Q^{2}>\mu^{2}$. Also noteworthy is the stability of $\bar{u}\left(x, Q^{2}\right)$ at $Q^{2} \gg \mu^{2}$, i.e. not only the perturbative LO/NLO one but also that with respect to our GRV(94) results. This stability is almost as good as the one required for a physical quantity like $F_{2}\left(x, Q^{2}\right)$. The situation is, as usual [3], different for $g\left(x, Q^{2}\right)$ which is, however, not as relevant since the gluon density is not directly measurable. In fact, despite the sizeable difference of the LO and NLO gluon distributions in Fig. 5 in the small- $x$ region, the directly measurable gluon-dominated heavy quark contribution $F_{2}^{c}\left(x, Q^{2}\right)$ shows a remarkable perturbative stability even for very large values of the (factorization) scale, such as $\mu_{F}^{2} \sim Q^{2}$, as will be shown in the next Section. Furthermore, $x g$ and $x \bar{u}$ at $Q^{2} \gg \mu^{2}$ increase almost linearly for $10^{-5} \lesssim x \lesssim$ $10^{-3}$ and $10^{-5} \lesssim x \lesssim 10^{-2}$, respectively, on the double-logarithmic plots in Fig. 5. They can thus effectively be represented by $x f\left(x, Q^{2}\right) \sim x^{-\lambda_{f}\left(x, Q^{2}\right)}$ with effective slopes

$$
\begin{array}{lll}
\lambda_{g}\left(x, 5 \mathrm{GeV}^{2}\right) \simeq 0.24(0.34), & \lambda_{g}\left(x, 20 \mathrm{GeV}^{2}\right) \simeq 0.30(0.39) \\
\lambda_{\bar{u}}\left(x, 5 \mathrm{GeV}^{2}\right) \simeq 0.21(0.19), & \lambda_{\bar{u}}\left(x, 20 \mathrm{GeV}^{2}\right) \simeq 0.26(0.27),
\end{array}
$$

in $\mathrm{NLO}(\mathrm{LO})$, valid in the above mentioned $x$-intervals. These steep $\left(\lambda_{f}>0\right)$ dynamical small- $x$ predictions are somewhat smaller than the ones of GRV(94) [3].

Fig. 6 is an alternative way to present our dynamical (steep) small- $x$ predictions using the slope of $F_{2}^{p}\left(x, Q^{2}\right)$. This directly measurable gluon dominated quantity, $d F_{2}^{p} / d \ln Q^{2}$, exhibits again a good perturbative stability for $Q^{2} \gtrsim 1 \mathrm{GeV}^{2}$. The similarity of our new (modified) results with our previous NLO GRV(94) ones [3] is interesting as well. We refrain here from plotting any experimental results which are usually $Q^{2}$-averaged [33, 34] and thus may easily give rise to erroneous and misleading interpretations and conclusions when compared with theoretical results for $d F_{2}^{p}\left(x, Q^{2}\right) / d \ln Q^{2}$ which depend strongly on the specific choices of $x$ and $Q^{2}$. Nevertheless, our slopes in Fig. 6 are consistent with present HERA measurements [33, 34] for not too small values of $Q^{2}$. 


\section{The role of further deep inelastic and hard scattering data and very small- $x$ predictions}

As mentioned in the Introduction and the previous Section, fixed-target DIS measurements and data on $F_{2}^{c}\left(x, Q^{2}\right), p p(n) \rightarrow \mu^{+} \mu^{-} X$ and the $p \bar{p} \rightarrow W^{ \pm} X$ charge asymmetry are relevant for fixing and testing $g\left(x, Q^{2}\right), \bar{d}-\bar{u}$ and $d / u$. In Figs. 7 and 8 we compare our fitted LO and NLO results with the fixed-target $F_{2}^{p}$ data [17-21] where we show only the kinematic region which has been used to determine our valence-like gluon and sea $(\bar{u}+\bar{d})$ and the valence input distributions at $Q^{2}=\mu_{\mathrm{LO}, \mathrm{NLO}}^{2}$ as given in eqs. (5) and (6). The quality of our NLO fits (which are slightly favored over their LO counterparts) in Fig. 7 clearly demonstrates that our chosen value of $\alpha_{s}\left(M_{Z}^{2}\right)=0.114$ in Section 2 is fully consistent with all present fixed-target high precision DIS (non-singlet) data in the large $-x$ region. In our present analysis we employ the $F_{2}^{n} / F_{2}^{p}$ data [22, 23] as extracted without any (still uncertain) corrections for a possible EMC effect and nuclear shadowing in deuterium. Together with the $d_{v} / u_{v}$ constraints from $\nu p$ and $\nu d$ data [24], this procedure leads to a 'traditional' large $-x$ behavior, $d_{v} / u_{v} \rightarrow 0$ as $x \rightarrow 1$. Note that we investigated more complicated parametrizations for $d_{v}\left(x, \mu^{2}\right)$ than used in (5) and (6), but found them unnecessary even at the present level of accuracy.

In Fig. 9 we compare our new (modified) LO and NLO parton distributions with the relevant data on DIS charm production [36, 37, 38, 39]. From HERA only the most recent (preliminary) 1997 ZEUS results [39] are displayed, since they supersede the previous (published) measurements [37, 38] by their greatly improved accuracy. As discussed in the previous Section, the gluon $g\left(x, \mu_{F}^{2}\right)$ dominated $F_{2}^{c}\left(x, Q^{2}\right)$ is calculated using the fully predictive fixed order ( $\mathrm{LO} / \mathrm{NLO})$ perturbation theory [3, 30] which also underlies the actual analysis of the partial data [37, 38] utilized for extracting the total $F_{2}^{c}\left(x, Q^{2}\right)$. The factorization scale $\mu_{F}$ (being as usual assumed equal to the renormalization scale) should be preferably chosen to be $\mu_{F}^{2}=4 m_{c}^{2}$ [29]. The resulting predictions in Fig. 9 are in perfect agreement with all available data (including the original fixed-target EMC data [36]) and are furthermore perturbatively stable. Even choosing a very large scale like

$\mu_{F}^{2}=4\left(Q^{2}+4 m_{c}^{2}\right)$, the NLO results remain essentially unchanged at small- $x$ [40] as

\footnotetext{
${ }^{3}$ The normalizations of the $F_{2}^{p}$ data sets are allowed to float within their experimental uncertainties; the resulting normalization factors are 1.00 (SLAC), 0.98 (BCDMS), 1.01 (NMC, E665). The BCDMS data have been taken as analyzed in [19], i.e., with a shift of the central values due to the main systematic error and correspondingly reduced full errors. This is still the consistent treatment, also in conjunction with the new NMC data [19]. Furthermore we take over the target-mass corrections of 19] for the SLAC and BCDMS $F_{2}^{p}$ data.
} 
shown by the 'high scale' dotted curves in Fig. 9. This latter stability renders attempts to resum supposedly large logarithms $\left(\ln Q^{2} / m_{c}^{2}\right)$ in heavy quark production cross sections superfluous [11, 12, 41]. It should be noted that the charm (and bottom) production data strongly constrain the gluon distribution and will eventually be used to determine $g\left(x, \mu_{F}^{2}\right)$ directly from experiment [8].

The asymmetry measurements of Drell-Yan dilepton production in $p p$ and $p d$ collisions 99, 10 which have been instrumental in fixing $\bar{d}-\bar{u}$ (or $\bar{d} / \bar{u}$ ), in particular the very recent Fermilab-E866 data [10] for the extented $x$ range $0.03 \lesssim x \lesssim 0.35$, are compared with our updated LO and NLO results in Fig. 10. For comparison the consequences of our NLO GRV(94) $\bar{d} / \bar{u}$ ratio are shown as well which has been originally constrained just by the CERN-NA51 measurement [9] at $x=0.18$. Our present new results for $\bar{d} / \bar{u}$ are also consistent with (although slightly lower than) the recent preliminary semiinclusive HERMES measurements [42] at $0.05 \leq x \leq 0.2$. The sensitivity of the Drell-Yan asymmetry on $\bar{d}-\bar{u}$ can be most easily seen from the LO expression

$$
A_{D Y} \equiv \frac{\sigma^{p p}-\sigma^{p n}}{\sigma^{p p}+\sigma^{p n}}=\frac{2 \sigma^{p p}}{\sigma^{p d}}-1=\frac{(u-d)(\bar{u}-\bar{d})+\frac{3}{5}(u \bar{u}-d \bar{d})}{(u+d)(\bar{u}+\bar{d})+\frac{3}{5}(u \bar{u}-d \bar{d})+\frac{4}{5} s \bar{s}}
$$

due to $\sigma^{p N} \propto \sum_{u, d, s} e_{q}^{2}\left[q\left(x_{1}\right) \bar{q}\left(x_{2}\right)+q\left(x_{2}\right) \bar{q}\left(x_{1}\right)\right]$. The relevant NLO differential Drell-Yan cross section $\sigma^{p N} \equiv d^{2} \sigma^{p N} / d M_{\mu^{+} \mu^{-}} d x_{F}$ can be found in the Appendix of [43, except for eq. (8a) which has to be modified [44, 45] in order to conform with the usual $\overline{\mathrm{MS}}$ convention for the number of gluon polarization states $2(1-\varepsilon)$ in $4-2 \varepsilon$ dimensions.

Having fixed $\bar{d}-\bar{u}$ and $\bar{d}+\bar{u}$ as well as the valence densities $u_{v}$ and $d_{v}$ (cf. Figs. 7 and 8), our strongly constrained $u$ and $d$ distributions are now confronted in Fig. 11 with the $W^{ \pm} \rightarrow \ell^{ \pm} \nu$ charge asymmetry measurements at the Fermilab $p \bar{p}$ collider [46]. The $W^{ \pm}$rapidity asymmetry

$$
A\left(y_{\ell}\right)=\frac{d \sigma\left(\ell^{+}\right) / d y_{\ell}-d \sigma\left(\ell^{-}\right) / d y_{\ell}}{d \sigma\left(\ell^{+}\right) / d y_{\ell}+d \sigma\left(\ell^{-}\right) / d y_{\ell}}
$$

of the charged leptons from the $W^{ \pm} \rightarrow \ell^{ \pm} \nu$ decays with the lepton rapidity $y_{\ell}$ is a sensitive probe of the difference between $u$ and $d$ quark distributions at $Q^{2}=M_{W}^{2}$. Our LO and NLO predictions are in perfect agreement with present data. A good agreement is also obtained 47] with our GRV(94) densities [3], as shown in Fig. 11, and even with our previous $(\bar{u}=\bar{d})$ dynamical LO and NLO distributions [1, 2].

Further constraints on the gluon distribution, besides those from DIS and Drell-Yan data [12], could be obtained via $p p \rightarrow \gamma X, p p \rightarrow$ jet $+X$, etc., but the predicted cross 
sections are quite sensitive to assumptions concerning the magnitude of the intrinsic transverse momentum $k_{T}$ of the partons which is not well understood at present. This holds true in particular for the prompt photon production data where, despite the large scale $\left(\mu_{R, F}\right)$ uncertainty 48, 49, an additional sizeable $k_{T}$-smearing seems to be required [49, 50, 11] in particular in view of the recent Fermilab (E706) $p B e \rightarrow \gamma X$ measurements [51]. A further interesting source of information, sensitive to $g\left(x, Q^{2}\right)$, will be provided by the longitudinal structure function $F_{L}\left(x, Q^{2}\right) \equiv F_{2}-2 x F_{1}$ as illustrated for example in [3]. The available data on $F_{L}$ are unfortunately still of rather limited accuracy [8, 52]. Finally it should be mentioned that the gluon density is also tested in a NLO determination of the strange sea quark density $s\left(x, Q^{2}\right)$ from neutrino induced charm production data, i.e. opposite-sign dimuon events originating from $\nu N \rightarrow \mu^{-} c X$ with $c \rightarrow \mu^{+} \bar{\nu}_{\mu} s$. Since dimuon events give, among other things, direct access to $s\left(x, Q^{2}\right)$ via $W^{+} s \rightarrow c, W^{+} s \rightarrow c g$ and $W^{+} g \rightarrow c \bar{s}$, etc., they also probe our purely dynamically generated $s\left(x, Q^{2}\right)$, i.e. the assumed vanishing strange sea input in eqs. (5) and (6), throughout the entire $x$-region. Since our slightly modified input densities in (5) and (6) give rise to $s\left(x, Q^{2}\right)$ and $g\left(x, Q^{2}\right)$ which are similar to the GRV(94) ones, they result in similarly agreeable predictions for $\stackrel{(-)}{\nu} N \rightarrow \mu^{-} \mu^{+} X$ [28]. It should, however, be kept in mind that a vanishing strange sea input is by no means a crucial ingredient of the dynamical approach.

As our parameter-free small- $x$ predictions for parton distributions at $x<10^{-2}$ are entirely of QCD-dynamical origin and depend, apart from intrinsic theoretical uncertainties, rather little on the detailed input parameters at $x \gtrsim 10^{-2}$, it is interesting to study these predictions in kinematic regions not accessible by present DIS experiments and to compare them with our previous $\operatorname{GRV}(94)$ densities. Of particular interest here is the comparison in Fig. 12 at $Q^{2}=10^{4} \mathrm{GeV}^{2}$ and extremely small $x$, i.e. $10^{-8} \lesssim x \lesssim 10^{-5}$, relevant to questions concerning neutrino astronomy [53]. These results at $x \lesssim 10^{-5}$ indicate that, for example, ultra-high-energy neutrino nucleon cross sections, which are sensitive to parton densities at $x$ values as small as $10^{-8}$, can be rather reliably calculated to within about $20 \%$. This follows not only from the perturbative stability at extremely small values of $x$, where moreover our improved predictions are comparable to the ones based on the NLO GRV(94) densities, but also from the fact that the predictions at $Q^{2} \simeq M_{W}^{2}$ are rather independent of the specific choice for the renormalization scale $\mu_{R}$ appearing in $\alpha_{s}\left(\mu_{R}^{2}\right)$ and for the factorization scale $\mu_{F}$ appearing in the parton densities $f\left(x, \mu_{F}^{2}\right)$. We have checked this by taking $\mu_{R}=\mu_{F}$ with [14, 32] $Q / 2 \leq \mu_{F} \leq 2 Q$ which requires, of 
course, also corresponding modifications $f$ in our input scale $\mu$ and $f\left(x, \mu^{2}\right)$ in eq. (6).

\section{Discussion and Summary}

As demonstrated above, the radiative (dynamical) GRV(94) parton distributions [3] disagree with recent precision HERA data only within the margins resulting from the $10 \%$ uncertainty in their input scale $\mu$ [2, 3]. Taking into account also the new large $-x$ parton constraints and $\alpha_{s}$-results, we have generated new sets of LO and NLO dynamical parton densities corresponding to $\alpha_{s}\left(M_{z}\right)=0.114$ for the purpose of future precision analyses. Nevertheless one can in practice still utilize the former GRV(94) distributions [3], in particular in view of the fact that in most applications of these parton densities to, say, high $-p_{T}$ jet, photon or heavy quark production, the usually considered (see, for example, 14, 32 for a recent review and comparative discussion) freedom in the choice of the factorization and/or renormalization scale, e.g. $p_{T} / 2 \lesssim \mu_{F, R} \lesssim 2 p_{T}$, overshadows the present modifications of our previous distributions [3].

It is also necessary to mention that the task of searching the ultimately correct parton distributions is not only affected by the above mentioned higher order uncertainties, but also by the discrepancies between the data sets used, e.g. between the NMC data [20] on the one hand and the CCFR [55] and HERA [6, 0, \&] data on the other, which are not well understood at present [33, 56]. Furthermore, recent attempts to calculate the (nonperturbative) input parton densities from first principles using the chiral soliton approach yielded, besides the valence densities, also a valence-like sea density in the small- $x$ region at $Q_{0}^{2}=0.3-0.4 \mathrm{GeV}^{2}$ - a scale set by the inverse average instanton size [57]. It remains to be seen, however, whether a sizeable valence-like gluon density at the same 'dynamical' input scale is also within the realm of this approach. It is also interesting to remark that a valence-like gluon input density, with a momentum fraction compatible with our results [1, 2, 3], has been obtained from considerations of intrinsic nucleon Fock-states [58].

Finally it should be emphasized that the stable parameter-free dynamical predictions for parton distributions in the extremely small- $x$ region, $10^{-8} \lesssim x \lesssim 10^{-5}$, allow for rather reliable estimates of ultra-high-energy neutrino nucleon cross sections relevant to questions concerning neutrino astronomy [53].

\footnotetext{
${ }^{4}$ The implementation of this modification amounts to replacing everywhere in 3, 54 the NLO $\alpha_{s}\left(Q^{2}\right)$ and the $f\left(x, Q^{2}\right)$ by $\alpha_{s}\left(\mu_{F}^{2}\right)$ and $f\left(x, \mu_{F}^{2}\right)$, respectively, while the common $\overline{\mathrm{MS}}$ Wilson coefficients in [3] have to be replaced by $C_{i} \rightarrow C_{i}+P_{q i}^{(0)} \ln Q^{2} / \mu_{F}^{2}$ for $i=q, g$.
} 
A FORTRAN package containing our new LO and $\mathrm{NLO}(\overline{\mathrm{MS}})$ parton densities as well as $F_{2}^{c, b}\left(x, Q^{2}\right)$, calculated in fixed-order perturbation theory, can be obtained by electronic mail on request. Instead of using the appropriate massive quark subprocesses for calculating heavy quark production rates in fixed-order perturbation theory, rough estimates (valid to within a factor of 2, say) of 'heavy' quark effects can be easier obtained with the help of the massless 'heavy' quark distributions $c\left(x, Q^{2}\right)$ and $b\left(x, Q^{2}\right)$ given in [1]. For further convenience we also provide the NLO(DIS) distributions which are related to the $\mathrm{NLO}(\overline{\mathrm{MS}})$ ones according to eq. (21) of ref. [3].

\section{Acknowledgements}

We thank S. Kretzer for several helpful numerical analyses, in particular for his cooperation in calculating the $W^{ \pm}$charge asymmetries in Fig. 11, as well as A. Milsztajn, L. Bauerdick, A. Bruell, E. Rondio, V. Shekelyan, M. Vincter, and R.G. Roberts for useful discussions and informations concerning recent data. This work has been supported in part by the "Bundesministerium für Bildung, Wissenschaft, Forschung und Technologie", Bonn. 


\section{References}

[1] M. Glück, E. Reya and A. Vogt, Z. Phys. C53 (1992) 127.

[2] M. Glück, E. Reya and A. Vogt, Phys. Lett. B306 (1993) 391.

[3] M. Glück, E. Reya and A. Vogt, Z. Phys. C67 (1995) 433.

[4] H1 Collab.: I. Abt et al., Nucl. Phys. B407 (1993) 515; T. Ahmed et al., ibid. B439 (1995) 471; S. Aid et al., Phys. Lett. B354 (1995) 494.

[5] ZEUS Collab.: M. Derrick et al., Phys. Lett. B316 (1993) 412; Z. Phys. C65 (1995) 379; Phys. Lett. B345 (1995) 576.

[6] H1 Collab.: S. Aid et al., Nucl. Phys. B470 (1996) 3; C. Adlofff et al., ibid. B497 (1997) 3.

[7] ZEUS Collab.: M. Derrick et al., Z. Phys. C69 (1996) 607; ibid. C72 (1996) 399.

[8] H1 Collab., Papers 260 and 275, submitted to the Int. Europhysics Conf. on HEP, Jerusalem, August 1997;

ZEUS Collab., Papers N-646 and N-647, ibid.

[9] A. Baldit et al., NA51 Collab., Phys. Lett. B332 (1994) 244.

[10] E.A. Hawker et al., E866 Collab., Phys. Rev. Lett. 80 (1998) 3715.

[11] A.D. Martin, R.G. Roberts and W.J. Stirling, Phys. Lett. B387 (1996) 419; A.D. Martin, R.G. Roberts, W.J. Stirling and R.S. Thorne, Univ. Durham DTP/98/10, RAL-TR-98-029 (hep-ph/9803445).

[12] H.L. Lai et al., CTEQ, Phys. Rev. D55 (1997) 1280;

J. Huston et al., CTEQ, FERMILAB-PUB-98/046T (hep-ph/9801444).

[13] Particle Data Group, R.M. Barnett et al., Phys. Rev. D54 (1996) 1;

M. Schmelling, MPI H-V39-1996 (hep-ex/9701002), Proceedings of the XXVIII Intern. Conf. on HEP, Warsaw, July 1996, eds. Z. Ajduk and A.K. Wroblewski (World Scientific 1997), p. 91;

S. Bethke, PITHA 97/37 (hep-ex/9710030), QCD Euroconference 97, Montpellier, Nucl. Phys. B (Proc. Supplement) 64 (1998) 54. 
[14] P.N. Burrows, SLAC-PUB-7293 (1996), (hep-ex/9612007), Proceedings of the 3rd Intern. Symp. on Radiative Corrections, Cracow, August 1996, eds. S. Jadach and M. Skrzypek, Acta Phys. Polon. B28 (1997) 701; P.N. Burrows et al., SLAC-PUB7371 (1996) (hep-ex/9612012), Proceedings of the 1996 DPF/DPB Summer Study on New Directions for HEP, Snowmass, June 1996, eds. D.G. Cassel et al. (SLAC 1997), p. 1096.

[15] W.T. Giele, FERMILAB-CONF-97/240-T (hep-ph/9707300), Proceedings of the 5th Intern. Workshop on DIS and QCD, Chicago, April 1997, eds. J. Repond and D. Krakauer (AIP, New York 1997), p. 414;

W.T. Giele and S. Keller, FERMILAB-PUB-98/082-T (hep-ph/9803393).

[16] P. Abreu et al., DELPHI Collab., CERN-PPE/97-108 (Z. Phys. C, to appear).

[17] L.W. Whitlow at al., Phys. Lett. B282 (1992) 475; L.W. Whitlow, SLAC-report-357 (1990).

[18] A.C. Benvenuti et al., BCDMS Collab., Phys. Lett. B223(1989) 485.

[19] A. Milsztajn and M. Virchaux, Phys. Lett. B274 (1992) 221; A. Milsztajn, private communication.

[20] M. Arneodo et al., NMC Collab., Nucl. Phys. B483 (1997) 3.

[21] M.R. Adams et al., E665 Collab., Phys. Rev. D54 (1996) 1006.

[22] M. Arneodo et al., NMC Collab., Nucl. Phys. B487 (1997) 3.

[23] A.C. Benvenuti et al., BCDMS Collab., Phys. Lett. B237 (1990) 599;

M.R. Adams et al., E665 Collab., Phys. Rev. Lett. 75 (1995) 1466.

[24] H. Abramowicz et al., CDHSW Collab., Z. Phys. C25 (1984) 29;

G.T. Jones et al., WA21 Collab., Z. Phys. C62 (1994) 601.

[25] G. Moreno et al., E605 Collab., Phys. Rev. D43 (1991) 2815.

[26] H. Abramowicz et al., CDHSW Collab., Z. Phys. C15 (1982) 19.

[27] CCFR Collab.: C. Foudas et al., Phys. Rev. Lett. 64 (1990) 1207; S.A. Rabinowitz et al., ibid. 70 (1993) 134; A.O. Bazarko et al., Z. Phys. C65 (1995) 189.

[28] M. Glück, S. Kretzer and E. Reya, Phys. Lett. B398 (1997) 381; B405 (1997) 392 (E). 
[29] M. Glück, E. Reya and M. Stratmann, Nucl. Phys. B422 (1994) 37.

[30] E. Laenen, S. Riemersma, J. Smith and W.L. van Neerven, Nucl. Phys. B392 (1993) 162.

[31] S. Riemersma, J. Smith and W.L. van Neerven, Phys. Lett. B347 (1995) 143;

K. Daum et al., Proceedings of the 1995/96 DESY-Workshop on the 'Future Physics at HERA', eds. G. Ingelmann, A. De Roeck and R. Klanner (DESY 1996), vol. 1, p. 89 .

[32] R.D. Ball and S. Forte, Phys. Lett. B358 (1995) 365;

J. Blümlein, S. Riemersma, W.L. van Neerven and A. Vogt, DESY 96-172 (hep-ph/9609217), Proceedings of the 1996 Zeuthen Workshop on Elementary Particle Theory, Rheinsberg, Germany, April 1996, eds. J. Blümlein, F. Jegerlehner and T. Riemann, Nucl. Phys. B (Proc. Suppl.) 51C (1996) 96.

[33] A. Caldwell, invited talk at the DESY Theory Workshop on 'Recent Developments in QCD', October 1997.

[34] H. Abramowicz and A. Levy, DESY 97-251 (hep-ph/9712415).

[35] J.E. Belz, HERMES Collab., Proceedings of the Symposium of Meson and Nucleon Interactions, Vancouver, Canada, August 1997 (to appear).

[36] J.J. Aubert et al., EMC Collab., Nucl. Phys. B213 (1983) 31.

[37] C. Adloff et al., H1 Collab., Z. Phys. C72 (1996) 593.

[38] J. Breitweg et al., ZEUS Collab., Phys. Lett. B407 (1997) 402.

[39] D. Bailey, ZEUS Collab., Proceedings of DIS '98, Brussels, April 1998 (to appear).

[40] A. Vogt, DESY 96-012 (hep-ph/9601352), Proceedings of DIS '96, Rome, April 1996, eds. G. D’Agostini and A. Nigro (World Scientific 1997), p. 254.

[41] M. Buza, Y. Matiounine, J. Smith and W.L. van Neerven, Phys. Lett. B411 (1997) 211.

[42] J. Ouyang, HERMES Collab., Proceedings of DIS '98, Brussels, April 1998 (to appear).

[43] P.J. Sutton, A.D. Martin, W.J. Stirling and R.G. Roberts, Phys. Rev. D45 (1992) 2349. 
[44] W. Furmanski and R. Petronzio, Z. Phys. C11 (1982) 293, and references therein.

[45] M. Glück, E. Reya and A. Vogt, Phys. Lett. B285 (1992) 285.

[46] F. Abe et al., CDF Collab., Phys. Rev. Lett. 74 (1995) 850;

A. Bodek, CDF Collab., Proceedings of the XXVIII Intern. Conf. on HEP, Warsaw, July 1996, eds. Z. Ajduk and A.K. Wroblewski (World Scientific 1997), p. 1438.

[47] S. Kretzer, E. Reya and M. Stratmann, Phys. Lett. B348 (1995) 628.

[48] W. Vogelsang and A. Vogt, Nucl. Phys. B453 (1995) 334.

[49] W. Vogelsang and M.R. Whalley, J. Phys. G23, Suppl. 7A (1997) A1.

[50] J. Huston et al., Phys. Rev. D51 (1995) 6139.

[51] L Apanasevich et al., E706 Collab., FERMILAB-PUB-97-351-E (hep-ex/9711017).

[52] C. Adloff et al., H1 Collab., Phys. Lett. B393 (1997) 452.

[53] T.K. Gaisser, F. Halzen and T. Stanev, Phys. Rep. 258 (1995) 173;

R. Gandhi, C. Quigg, M.H. Reno and I. Sarcevic, Astropart. Phys. 5 (1996) 81;

G.M. Frichter, D.W. McKay and J.P. Ralston, Phys. Rev. Lett. 74 (1995) 1508; talk given at the 7th Intern. Symp. on Neutrino Telescopes, Venice, Italy, 1996 astroph/9606008).

[54] M. Glück, E. Reya and A. Vogt, Z. Phys. C48 (1990) 471.

[55] CCFR Collab.: P.Z. Quintas et al., Phys. Rev. Lett. 71 (1993) 1307;

W.G. Seligman et al., ibid. 79 (1997) 1213.

[56] R. Devenish, Oxford report OUNP-98-01 (hep-ex/9801013), plenary talk given at the International Europhysics Conference on High-Energy Physics (HEP97), Jerusalem, August 1997.

[57] D. Diakonov, V. Petrov, P. Pobylitsa, M. Polyakov and C. Weis, Nucl. Phys. B480 (1996) 341; Phys. Rev. D56 (1997) 4069.

[58] P. Hoyer and D.P. Roy, Phys. Lett. B410 (1997) 63. 


\section{Figure Captions}

Fig. 1 Comparison of our NLO GRV(94) small- $x$ predictions [3] for the proton structure function $F_{2}^{p}$ with recent precision measurements at HERA [6, 6]. For illustration we also include the most recent preliminary data [8] (open symbols). The typical uncertainties of the GRV(94) predictions are illustrated by the dotted curves which are obtained by increasing $\mu_{\mathrm{NLO}}$ by $10 \%$, keeping everything else (valence-like input densities, $\alpha_{s}$, etc.) unchanged. The results of our present new analysis, where all these input quantities are consistently modified, lie in between the curves shown at small- $x$.

Fig. 2 As in Fig. 1, but for the NLO gluon density at $Q^{2}=20 \mathrm{GeV}^{2}$. The result of our present new analysis is shown as well (solid curve). The shaded bands represent the preliminary experimental small $-x$ constraints as extracted from $F_{2}$-measurements at HERA and the four data points are derived from deep-inelastic inclusive charmproduction as analyzed by $\mathrm{H} 1[8]$.

Fig. 3 Comparison of our new LO and NLO small- $x$ results for $F_{2}\left(x, Q^{2}\right)$, arising from the inputs (5) and (6), with HERA data for $Q^{2} \gtrsim 1 \mathrm{GeV}^{2}$ [6, 7, 8]. The valence-like NLO input, according to Fig. 4 , is shown by the curve $\left(\mu_{\mathrm{NLO}}^{2}\right)$ at the lower right corner. To ease the graphical representation we have plotted $F_{2}\left(x, Q^{2}\right)+i\left(Q^{2}\right) \times 0.5$, with $i$ indicated in the figure. The published data [6, 7] (closed symbols) are, different from Fig. 1, shown with small normalization changes as obtained in our fits: H1(94)*0.99, ZEUS(94)*1.01, shifted vertex data $[\mathrm{ZEUS}(94)$ and H1(95)]*0.97.

Fig. 4 The valence-like LO and NLO input densities $x f\left(f=u_{v}, d_{v}, \bar{u}, \bar{d}, g\right)$ according to eqs. (5) and (6) at $Q^{2}=\mu_{\mathrm{LO}}^{2}=0.26 \mathrm{GeV}^{2}$ and $Q^{2}=\mu_{\mathrm{NLO}}^{2}=0.40 \mathrm{GeV}^{2}$. The strange sea $s=\bar{s}$ vanishes at the input scales $Q^{2}=\mu_{\mathrm{LO}, \mathrm{NLO}}^{2}$. The NLO GRV(94) input [3] is also shown for comparison, as well as the evoluted results at $Q^{2}=5 \mathrm{GeV}^{2}$.

Fig. 5 The small- $x$ behavior of our radiatively generated gluon and sea-quark distributions in LO and NLO. The valence-like inputs, according to eqs. (5) and (6) as presented in Fig. 4, are shown by the lowest curves referring to $\mu^{2}$ for illustration. For comparison we also show the NLO GRV(94) predictions [3]. The results are multiplied by the numbers indicated in brackets.

Fig. 6 The predicted slope $d F_{2}^{p}\left(x, Q^{2}\right) / d \ln Q^{2}$ in LO and NLO. The difference between the dotted and solid curves is due to the NLO charm contribution $d F_{2}^{c}\left(x, Q^{2}\right) / d \ln Q^{2}$. For comparison we also show the NLO results of GRV(94) [3]. The upper part of the 
figure refers to the values of $Q^{2}$ appropriate to the HERA measurements [33, 34, 11], and the lower one to a representative fixed value of $Q^{2}$.

Fig. 7 Comparison of our LO and NLO fits with fixed-target $F_{2}^{p}$ data [17, 18, 19, 20, 21] in the $Q^{2} \geq 4 \mathrm{GeV}^{2}, W^{2} \geq 10 \mathrm{GeV}^{2}$ region which were used for determining the valence and valence-like gluon and sea input in (5) and (6). The data sets are shown with their normalization factors as obtained in the fit. Some points with large errors, e.g. E665 at large- $x$, are omitted.

Fig. 8 Same as in Fig. 7 but for the measured $F_{2}^{n} / F_{2}^{p}$ and $d_{v} / u_{v}$ ratios [22, 24]. Only the NMC results for $F_{2}^{n} / F_{2}^{p}$ are shown, as they are much more accurate than the corresponding BCDMS and E665 data [23]. The preliminary semi-inclusive HERMES data for $d_{v} / u_{v}$ are displayed as well [35]. For comparison our previous NLO GRV(94) results [3] are also shown.

Fig. 9 LO and NLO predictions for $F_{2}^{c}\left(x, Q^{2}\right)$ in fixed-order perturbation theory ( $\gamma^{*}$-gluon fusion, etc.) based on our new LO and NLO parton densities using $\mu_{F}^{2}=4 \mathrm{~m}_{c}^{2}$, $m_{c}=1.4 \mathrm{GeV}$, compared with data from EMC [36] and ZEUS [39]. The NLO results based on the NLO GRV(94) parton densities [3] are close to the present LO(NLO) curves at large (small) values of $x$. The NLO (high scale) curves refer to a significantly larger factorization scale, $\mu_{F}^{2}=4\left(Q^{2}+4 m_{c}^{2}\right)$.

Fig. $10 \mathrm{LO}$ and NLO QCD results for $\sigma^{p d} / 2 \sigma^{p p}$ for $A_{D Y}$ in eq. (8) compared with the NA51 [9] and the most recent E866 [10] Drell-Yan dimuon production data. The experimental (E866) normalization uncertainty of 0.01 has been used in the fit. The $\mathrm{NLO}(94)$ curves correspond to the $\bar{d} / \bar{u} \operatorname{GRV}(94)$ ratio [3] which has been originally extracted just from the the NA51 data point at $x=0.18$ shown in the right figure.

Fig. $11 \mathrm{LO}$ and NLO predictions for the $W^{ \pm}$charge asymmetry $A\left(y_{\ell}\right)$ in eq. (9). Note that these data have not been used for fixing our new input distributions. The NLO(94) predictions, based on the GRV(94) densities [3], are shown for comparison. The relevant LO/NLO expressions for $A\left(y_{\ell}\right)$ can be found in 47 (and references therein). The Fermilab-CDF data are taken from 46.

Fig. 12 Predictions for $F_{2}^{p}\left(x, Q^{2}\right)$ for extremely small values of $x$. The difference between the dotted and solid curves is due to the NLO heavy quark (charm, bottom) contributions, which derive from photon-gluon (quark) fusion processes. The NLO(94) results correspond to the parton densities of [3]. The NLO results at these large values of $Q^{2}$ are insensitive to the specific choice of the factorization scale $\mu_{F}$. 


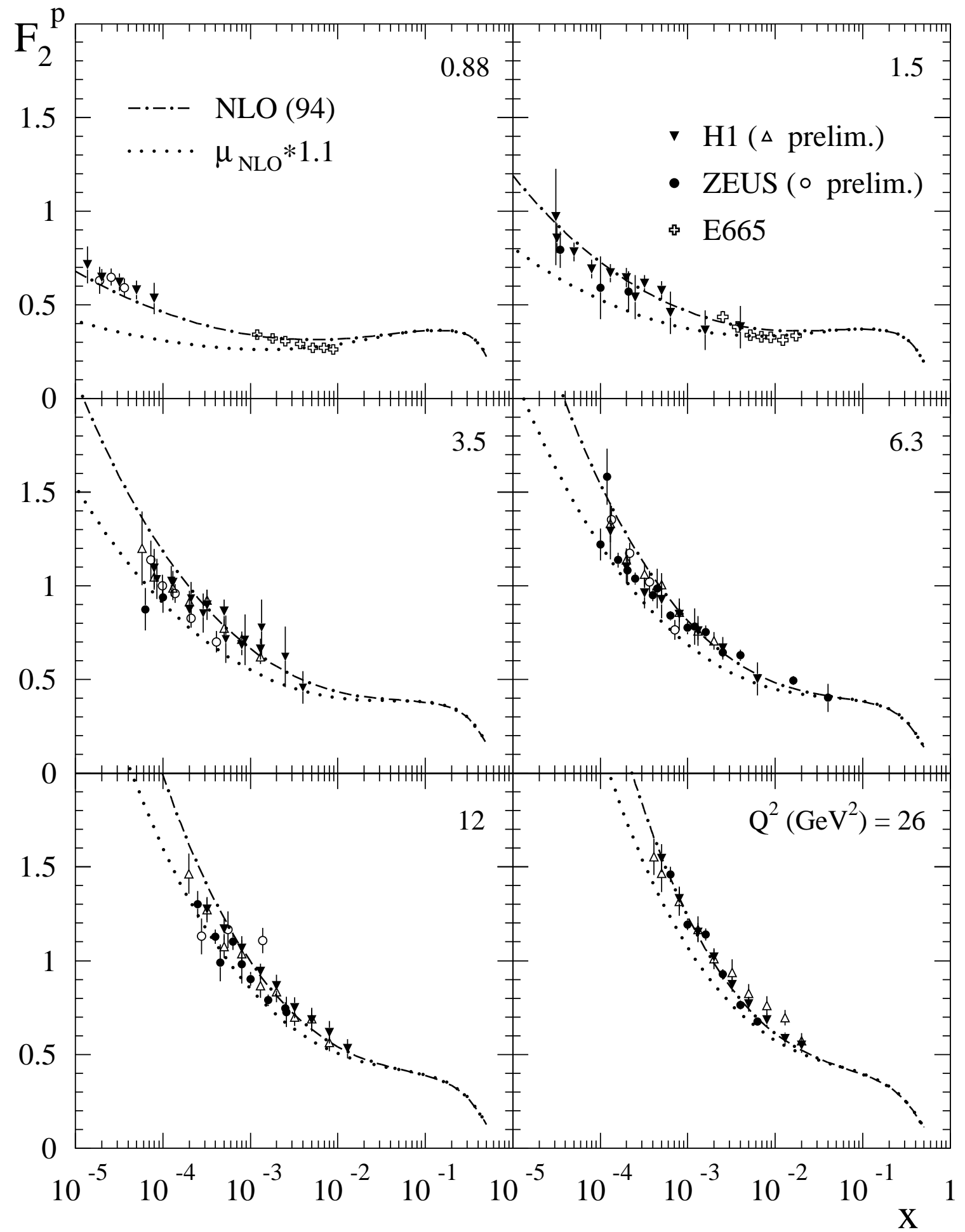

Fig. 1 


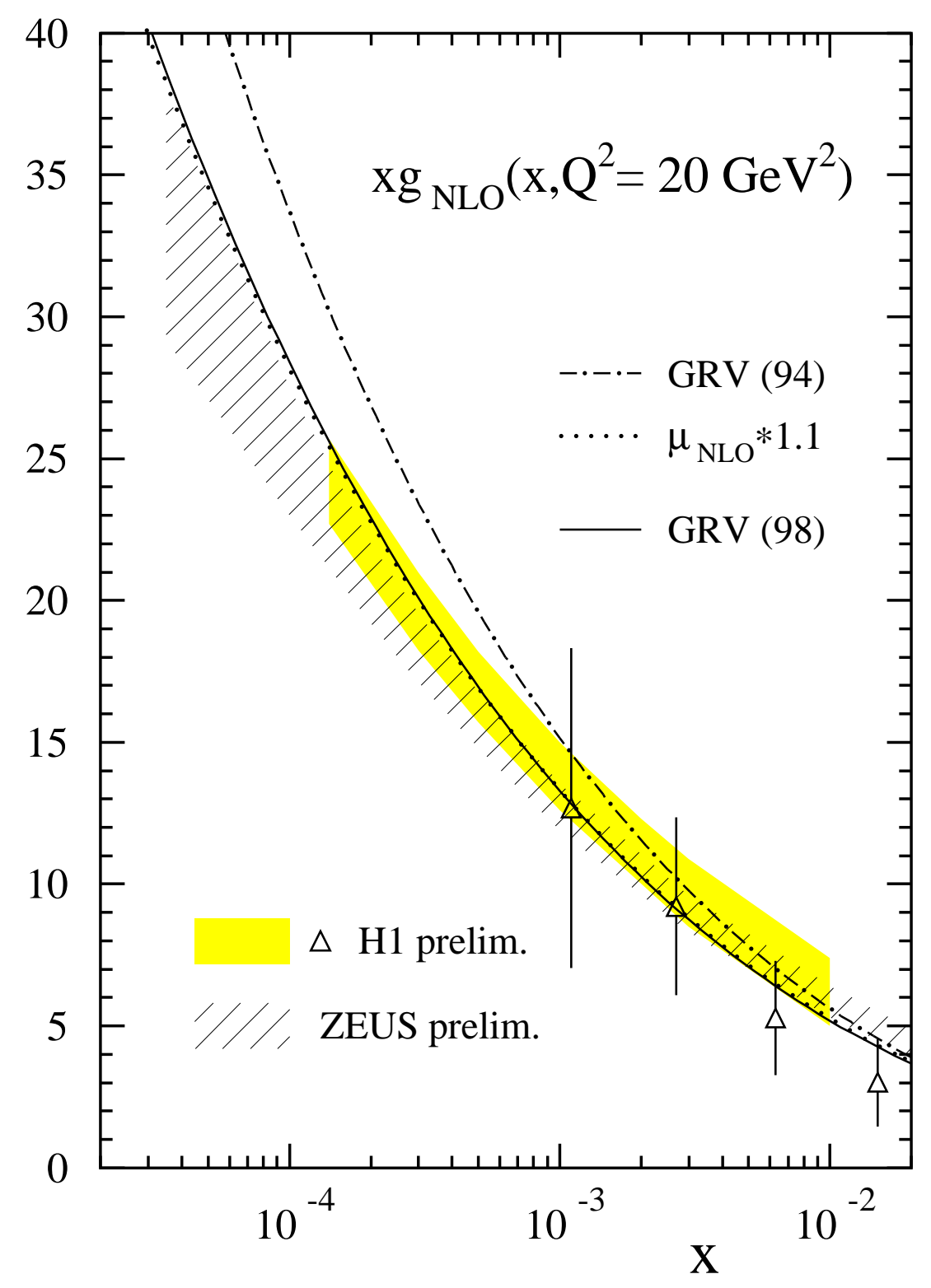

Fig. 2 

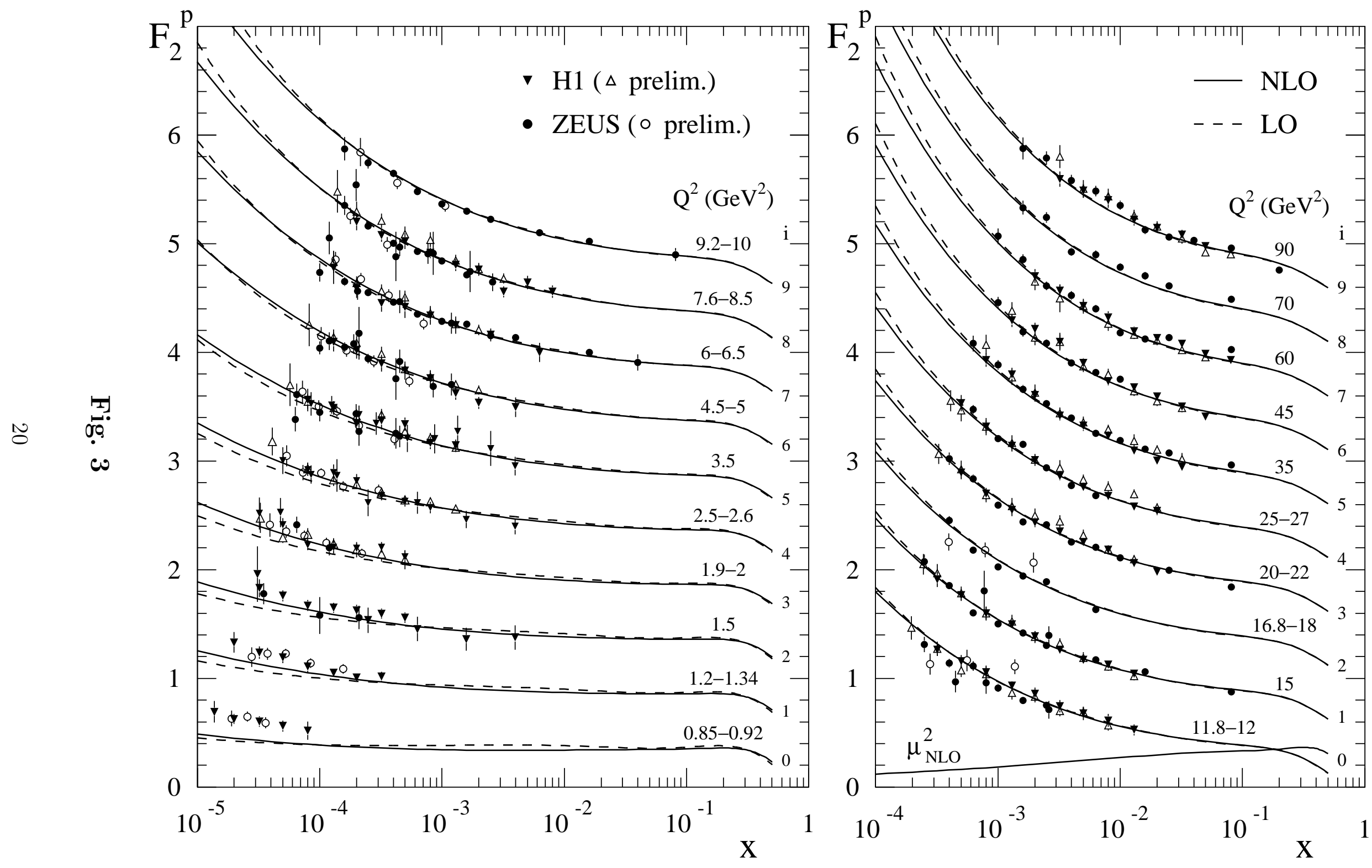


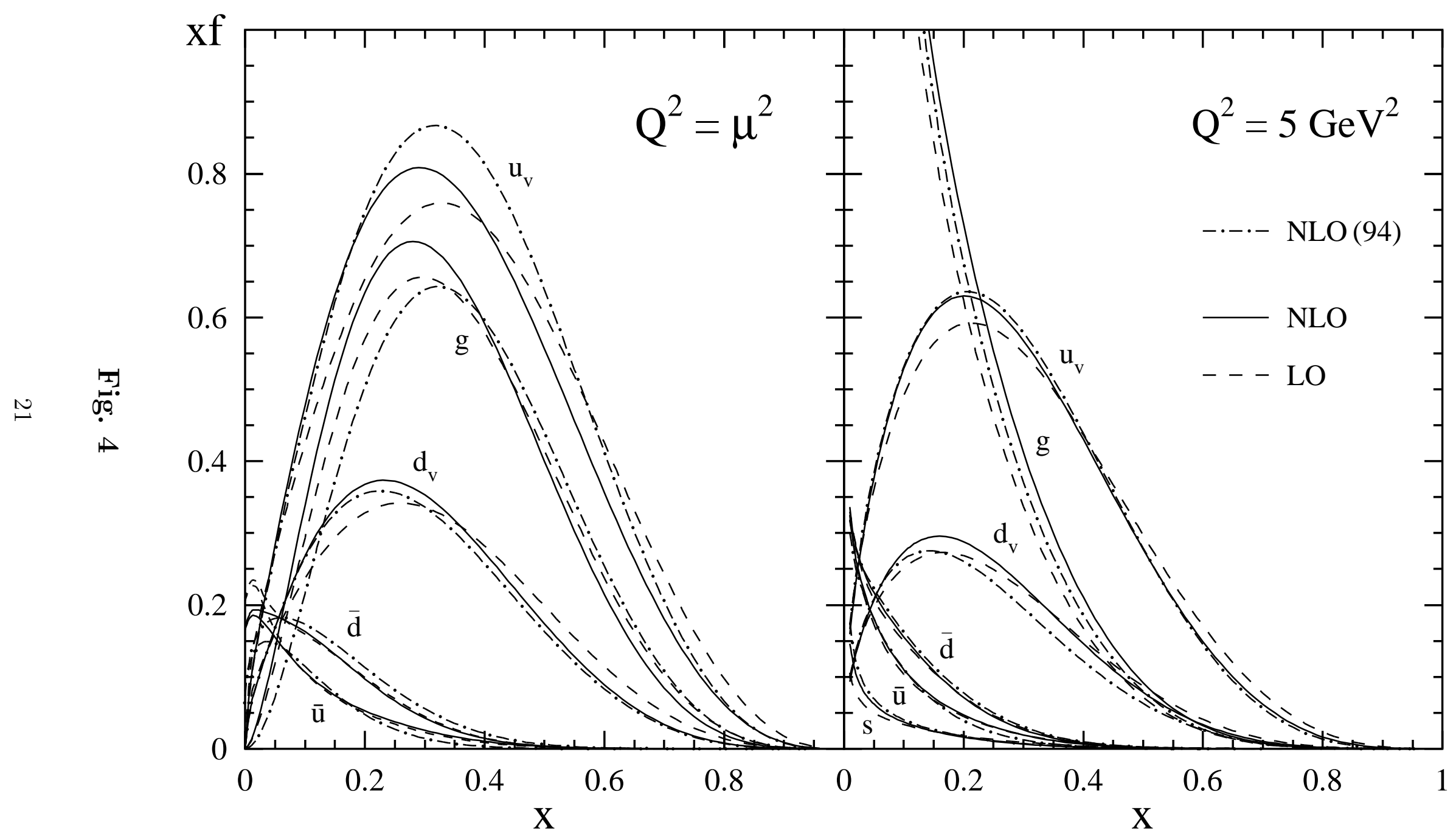



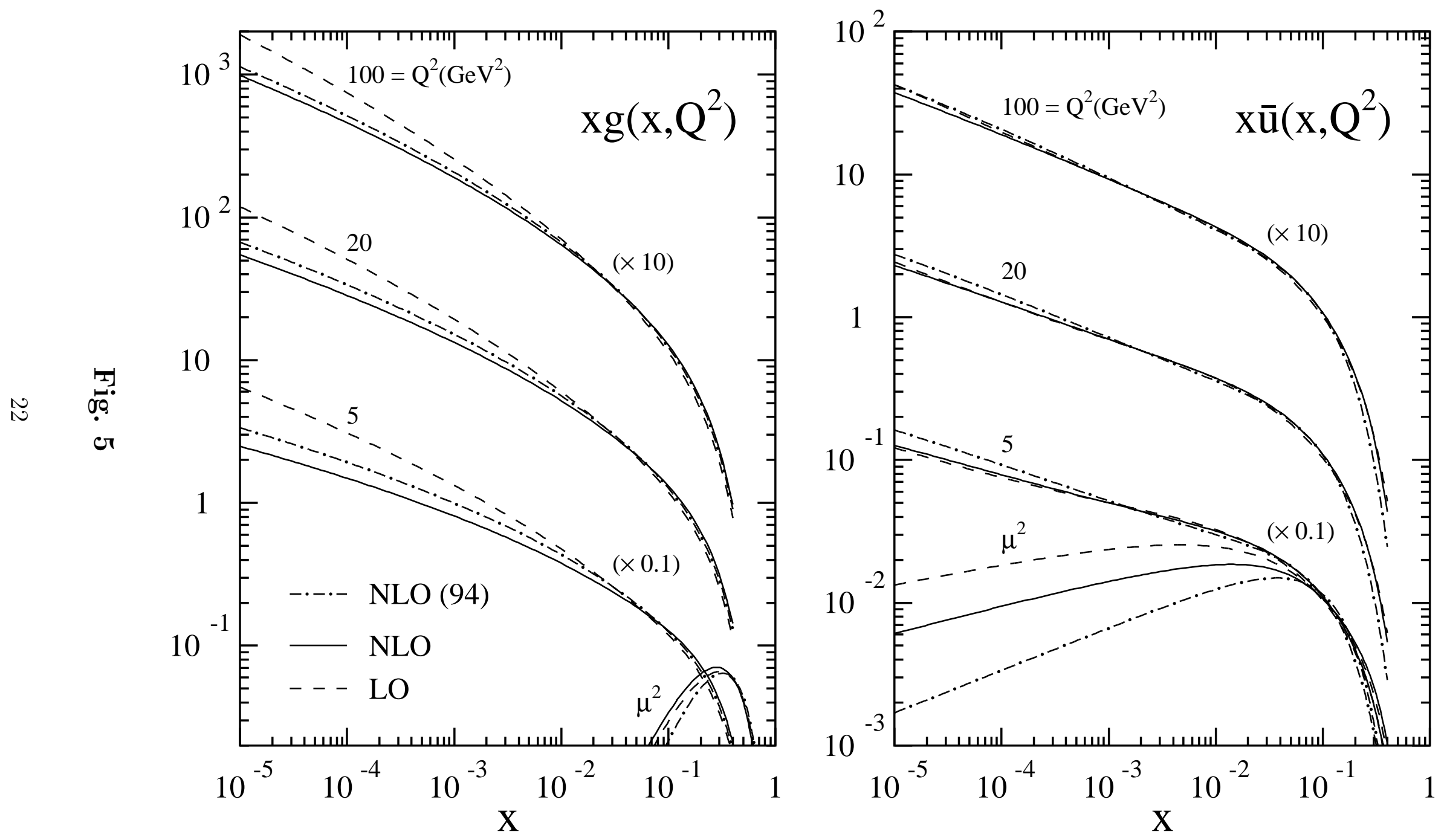


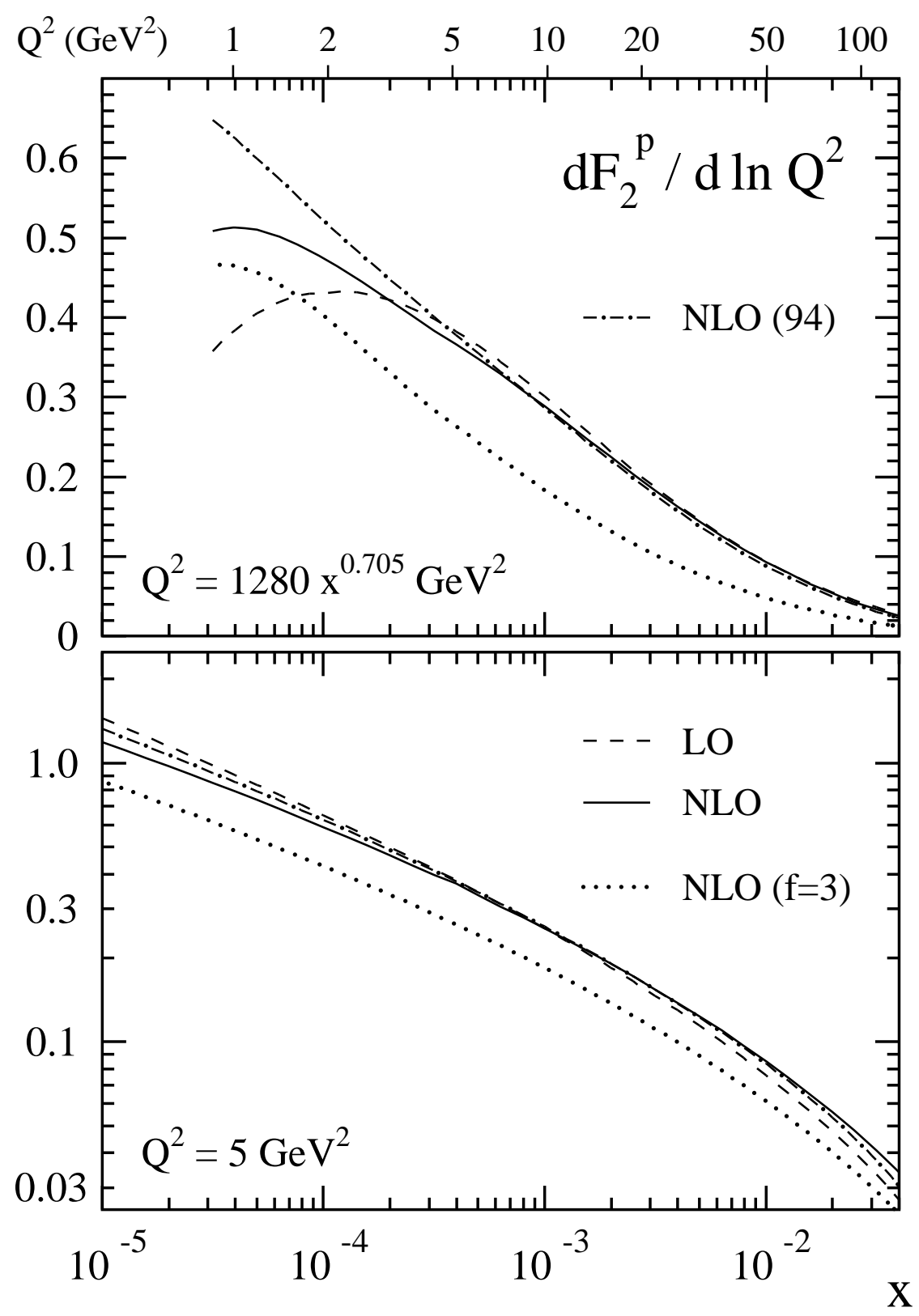

Fig. 6 

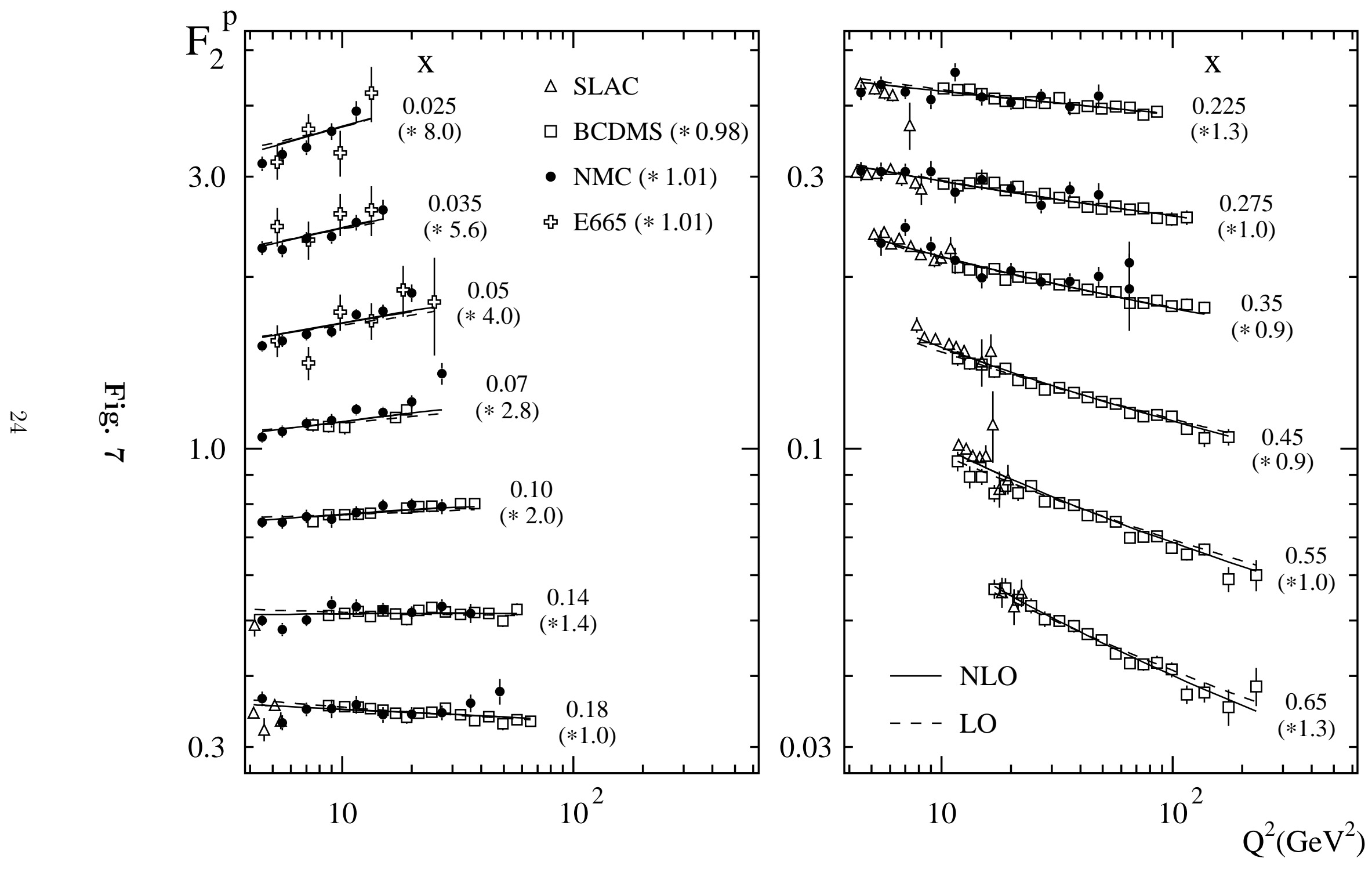

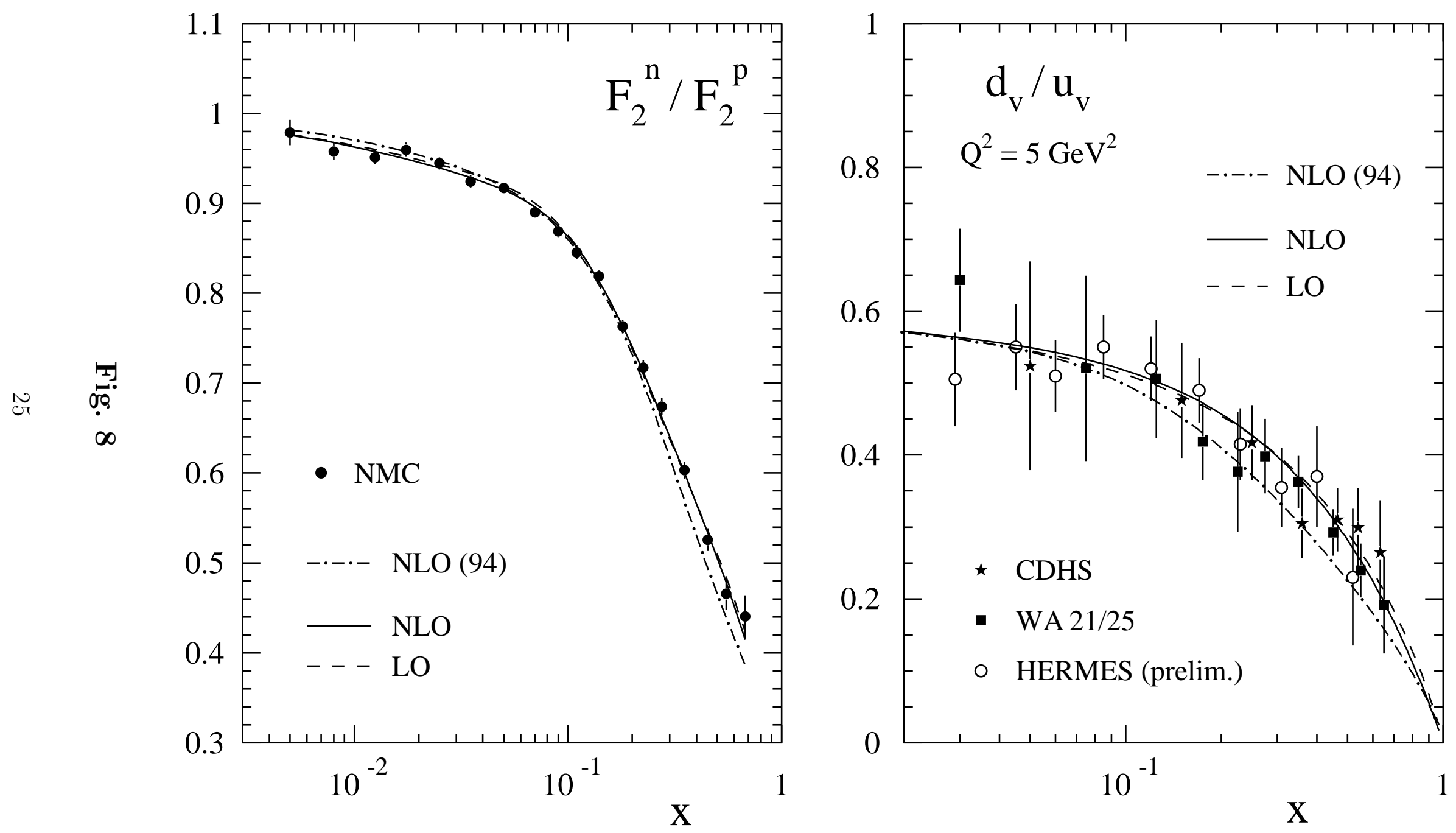


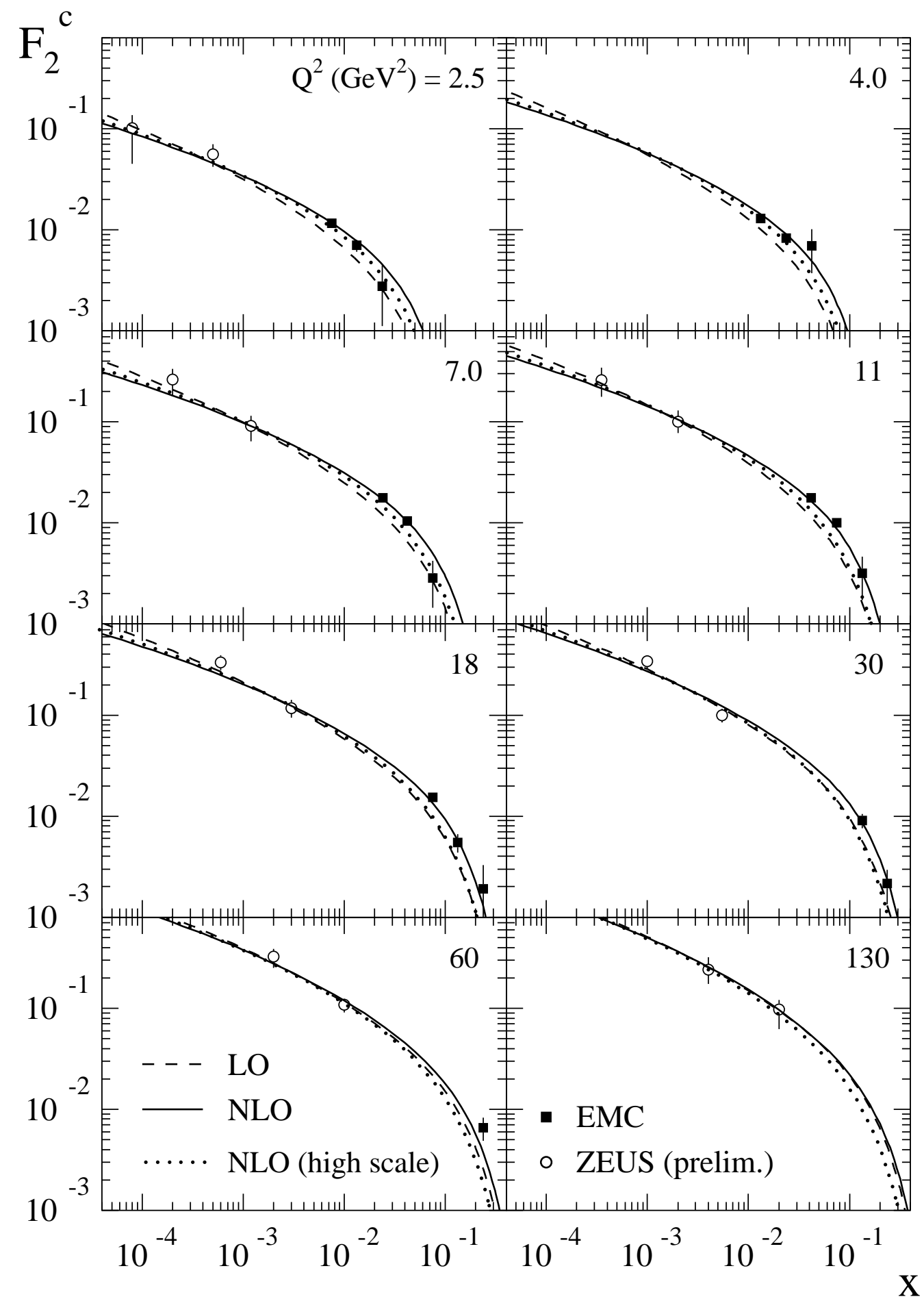

Fig. 9 

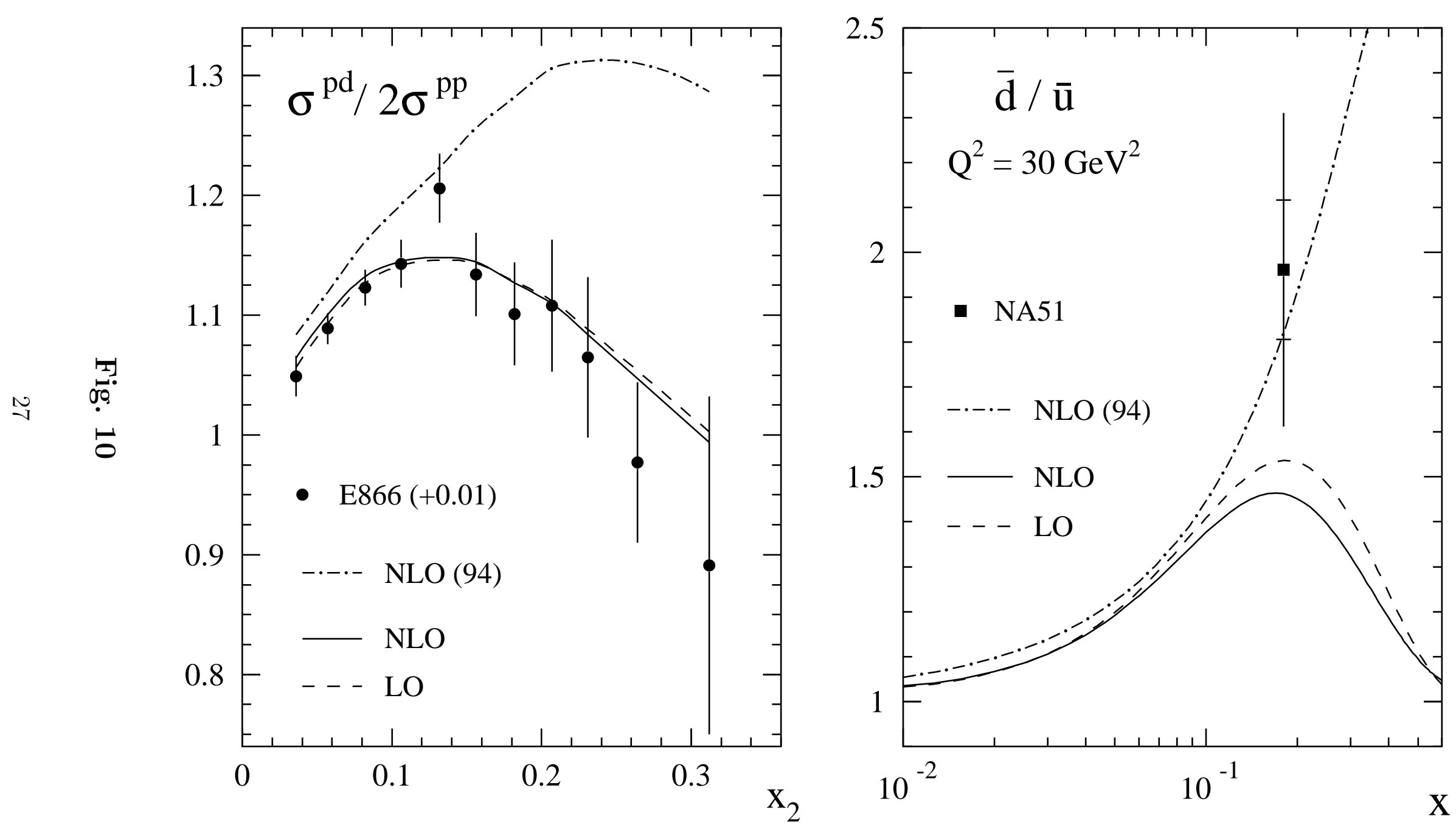


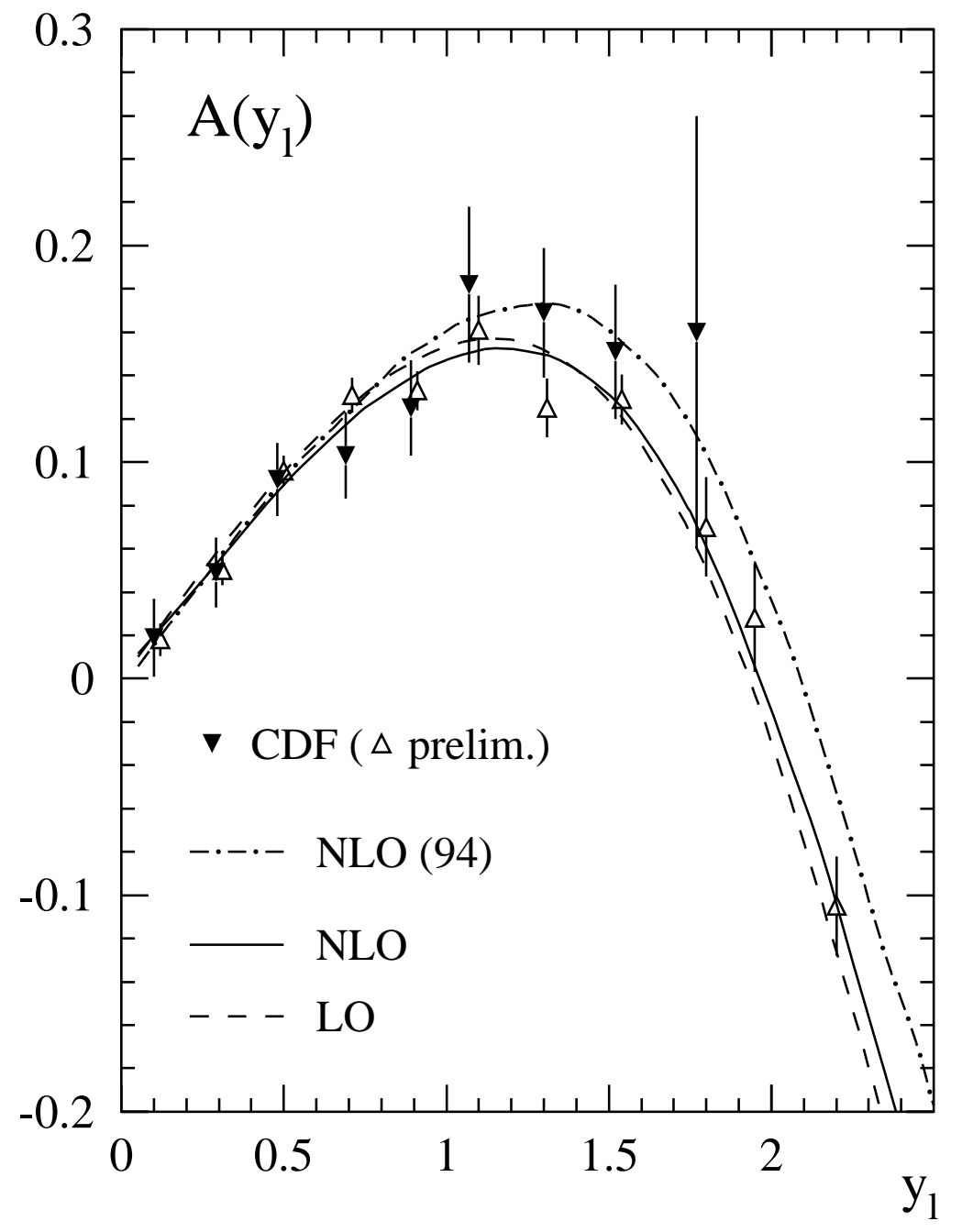

Fig. 11 


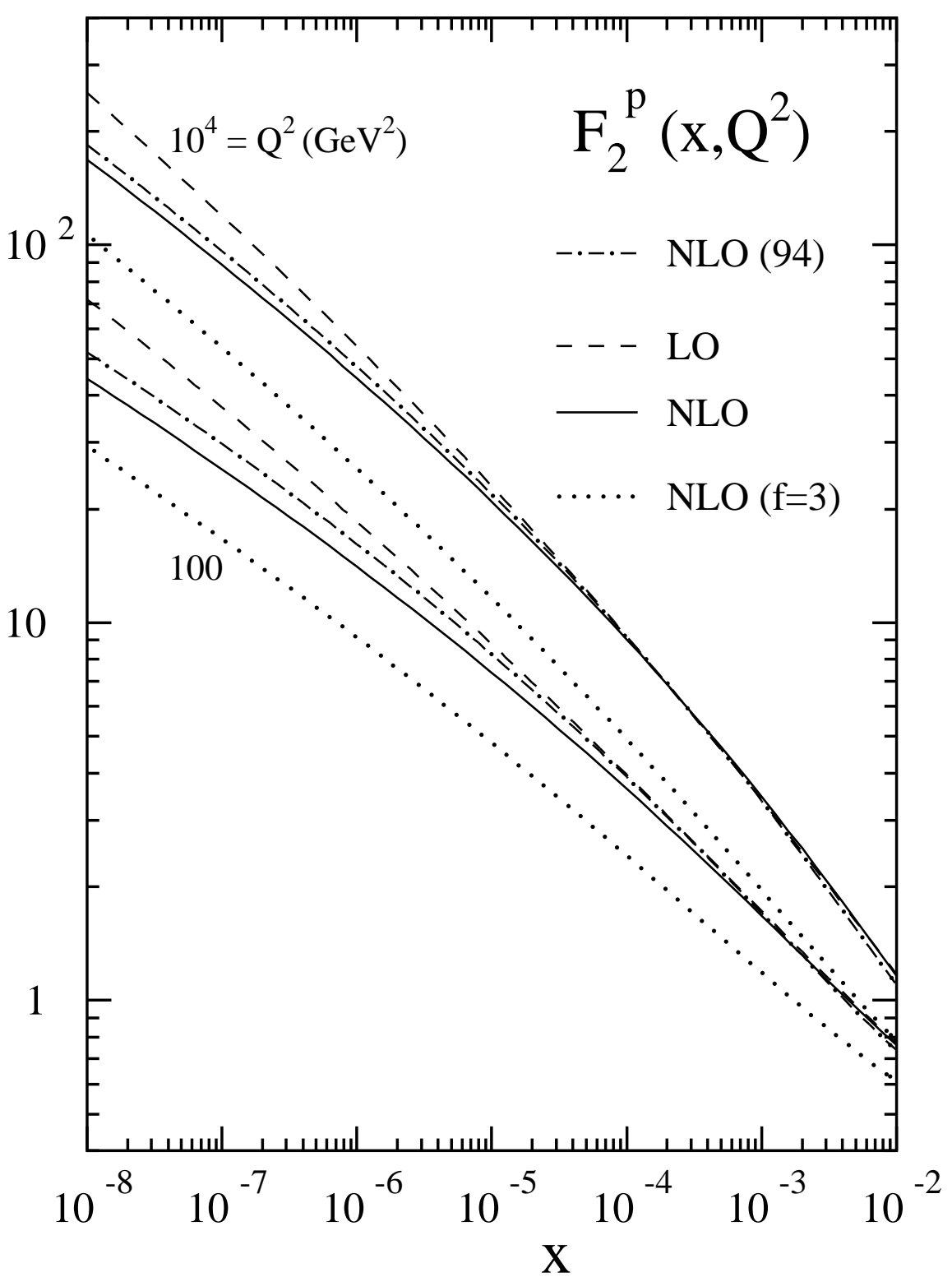

Fig. 12 